Beatrice Vogel ${ }^{1+2}$, Axel Heinemann ${ }^{1}$, Antonios Tzikas ${ }^{1}$, Chanasom Poodendaen $^{1}$,
Helmut Gulbins ${ }^{2}$, Hermann Reichenspurner ${ }^{2}$, Klaus Püschel ${ }^{1}$, Hermann Vogel ${ }^{1}$

\title{
Post-mortem computed tomography (PMCT) and PMCT-angiography after cardiac surgery. Possibilities and limits
}

\author{
1 Institute for Forensic Medicine, University Medical Center Hamburg-Eppendorf \\ 2 University Heart Center, Department of Cardiovascular Surgery, Hamburg
}

Background: PMCT is a well-known tool of the forensic pathologist. It is employed worldwide. PMCT-angiography offers additional insights. This paper intends to demonstrate possibilities of both methods after cardiac surgery.

Material and Methods: Exemplary cases with typical findings were selected from our own collection. PMCT was performed as whole body CT (1 mm slice, pitch 1.5, 130kV, 180-130mAs, 16 slice MDCT). In PMCT-angiography, contrast material (1.2 litres) is injected into the arteries (arterial phase, also documented with a whole body $\mathrm{CT})$. Thereafter, contrast material is injected into the veins (venous phase, also documented with a whole body CT). The final CT is obtained after circulation has been provoked with a special pump (circulatory phase).

Results: PMCT visualised pseudoarthrosis and fractures of the sternum, implanted valves (TAVI) encroaching the ostia of the coronary arteries, bleeding and pericardial tamponade. PMCT-angiography showed the sources of the bleeding, vascular stenosis and obstruction and modified vascular supply. With respect to the postoperative care, malposition of tubes, drainages and complication of punctures could be seen.

Conclusion: PMCT and PMCT-angiography can visualise complications and the cause of death. Such knowledge may allow for prevention of suffering and death. It may also aid in improving valve design and implantation procedures.

Key words:

post-mortem, CT, angiography, cardiac surgery

\section{BACKGROUND AND PURPOSE}

Multi-Detector Computed Tomography (MDCT) has become an important tool in many forensic institutes $[1,2,3,4,5,6,7,8,9,10,11]$. In Hamburg, it is part of the routine. Post-mortem Computed Tomography (PMCT) has become a standard part of the post-mortem examination; it shows additional details and guides the subsequent autopsy.

PMCT-angiography complements PMCT [12, $13,14,15,16,17,18,19,20]$. The value of both methods for analysing cardiac disease has been described [21, 22, 23, 24]. Considering that PMCT-angiography visualises the vessels and the heart, it seems self-evident that PMCT-angiography should also have the potential to visualise postoperative morphology, especially after cardiac surgery.

Former cooperation with intensive care specialists has produced results of value for the care of patients in critical condition [25]. This leads to reflections, whether PMCT and PMCT-angiography would allow for ananalysis of surgical "failures" - as to their value in differentiating between more life-threatening and less serious complications. Furthermore, it is of interest if both procedures are apt to exclude technical faults and human errors. From the beginning, it has been clear that such an approach had to take into account the special situation of the surgeon and other specialists involved: when a patient dies during or shortly after surgery, the surgeon and the intensive care specialist consider this a "failure". Consciously or unconsciously, they try to prevent reproach. This may result in limited and/or selected "protective" information provided to the pathologist. This makes quite a differ- 
ence for the post-mortem evaluation compared to the ante-mortem situation in clinical radiology: the clinical physician looks for a diagnosis or therapeutic options; therefore, he usually informs the radiologist without any bias. This difference has to be kept in mind when determining possibilities and limits of PMCT and PMCT-angiography. Another aspect has also to be considered: in recent years, recording an action for proving its correctness has become more and more obligatory [26]. PMCT and PMCT-angiography could meet this need.

All these items were considered when we defined the goal of this project: to determine all relevant findings provided by PMCT and PMCT-angiography after cardiac surgery.

\section{MATERIAL AND METHODS}

Our PMCT archive for teaching and demonstration was searched for findings following death after heart surgery - either directly related to this procedure or as a non-related incidental findings much later on. This archive concentrates on postoperative morphology in PMCT. Furthermore, the cases were also reviewed where PMCT-angiography had been done $(n=139)$.

Since 2008, some 4000 PMCT have been performed as a routine part of the PM examination: until 2012, with a 6-sclice MDCT (MX 8000, Philips), since 2013 with a 16-slice MDCT (Brilliance, Philips).

In Hamburg, PMCT-angiography is practiced according to the modification proposed by Grabherr et al [16, 17]: following the initial whole-body CT (slice thickness $1 \mathrm{~mm}$, pitch 1.5; 180-200mAs, $130 \mathrm{kV})$, oily contrast material is injected into an artery, mostly the femoral artery (1200 ml, stationary arterial phase), after which another whole-body CT is done. Thereafter, contrast material is injected into a vein $(1000 \mathrm{ml}$, mostly into the femoral vein, so-called "venous" phase), followed by a third whole-body CT. Finally, a special pump produces circulation, and this final whole-body CT documents a circulatory vascular phase, the so called "circulation phase"). During these different phases the head, the heart, and the intestine may be examined with additional CT of high resolution (slice thickness $0.8 \mathrm{~mm}$, pitch $1.0,180-230 \mathrm{mAs}, 130 \mathrm{kV}$ ), if the clinical information available may mandate this.
By the way, the oily contrast material does not irritate the wall of arteries and veins and therefore does not limit later histology.

The deceased are from Hamburg and the neighbouring federal states. Formal consent had been obtained for PMCT and PMCT-angiography. Subsequent additional autopsy was performed in individual cases.

\section{RESULTS}

The findings primarily involve the sternum and the rib cage, the heart and its contiguous large vessels, and postoperative intensive care.

\section{Pseudoarthrosis and Fractures}

The sternum showed several forms of pathology: a pseudoarthrosis (resulting from the median osteotomy) involved the whole sternum or just the manubrium only (Fig. $1 \mathrm{a}$ and b). A dehiscence of the two halves of the bone after extraction of the wire-sutures indicated discomfort and possible additional complications (Fig. 2). This was also the case when the bone in the proximity of the osteotomy showed signs of non-infectious irritation, or if a wire-suture had migrated through one-half of the bone. These findings naturally appeared long after surgery and were usually incidental findings in not directly cardiac-surgery-related PM examinees. Close to the time of surgery, however, and also long time afterwards, there were non-sternotomy-related (but reanimation-induced) sternal and rib fractures, both of the bony and/or cartilaginous portions.

\section{Bleeding}

Bleeding was usually a serious complication observed in connection with different surgical procedures. PMCT showed blood in the chest (Fig. 4a) and the mediastinum. Cardiac tamponade was a special form of bleeding. PMCT-angiography localised the bleeding vessel (Fig. $4 \mathrm{~b}$ and $\mathrm{c}$ ). Bleeding could originate from one vessel and/or from several vessels (Fig. $4 \mathrm{~b}$ and $\mathrm{c}$ ). The latter was attributed to coagulation disorders. A singular observation showed contrast material in the myocardium, indicating the cardiotomy site in TAVI (Fig. 5); contrast material did not enter the pericardium, though. 

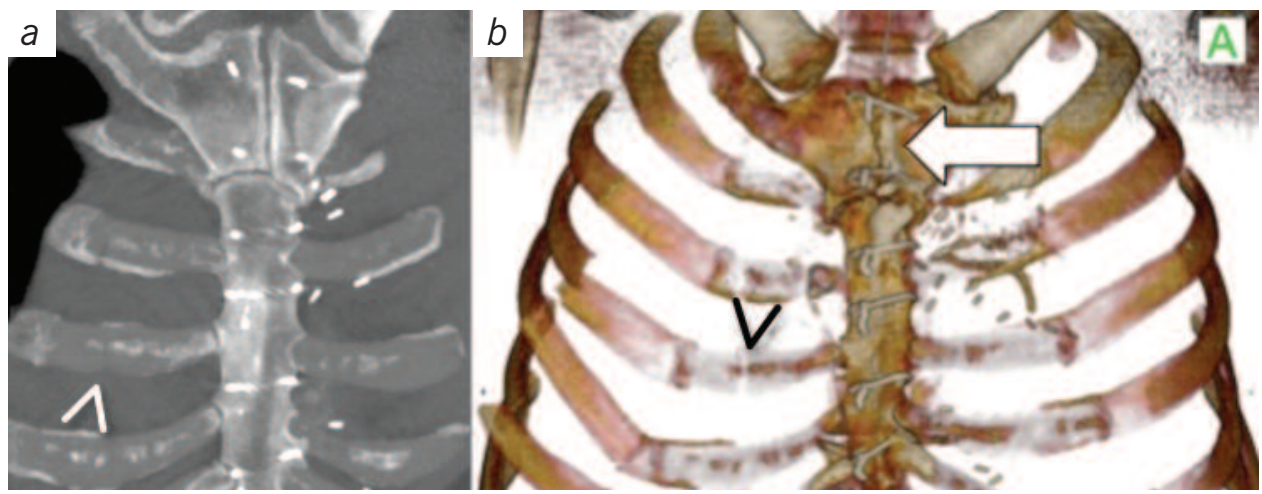

Fig. 1a and b. Pseudoarthrosis of the manubrium (arrow), many years after LIMA (Left Internal Mammary Artery) bypass (clips in typical position). Fracture of right ribs 4-6, and left ribs 4-6, and fractured cartilage of the $3^{\text {rd }}$ and $4^{\text {th }}$ right rib (arrow) due to recent reanimation. a: MIP (Maximum Intensity Projection. b: 3D reconstruction.

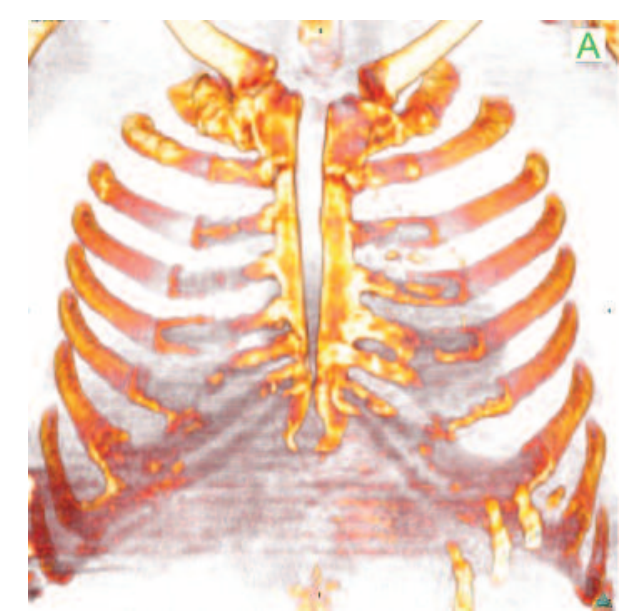

Fig. 2. Dehiscence of the manubrium and corpus sterni. Extraction of wire-sutures, many years after cardiac surgery. 3D reconstruction.

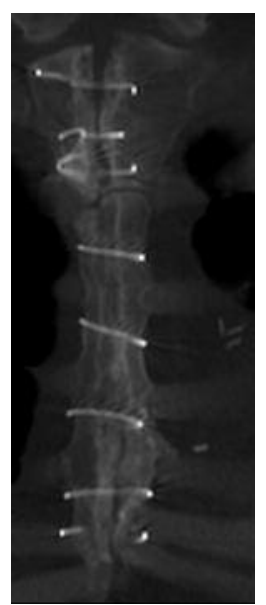

Fig. 3. Pseudoarthrosis of the whole sternum and signs of non-infectious reactive sclerosis of the bone adjacent to the osteotomy. Many years after cardiac surgery. MIP. 

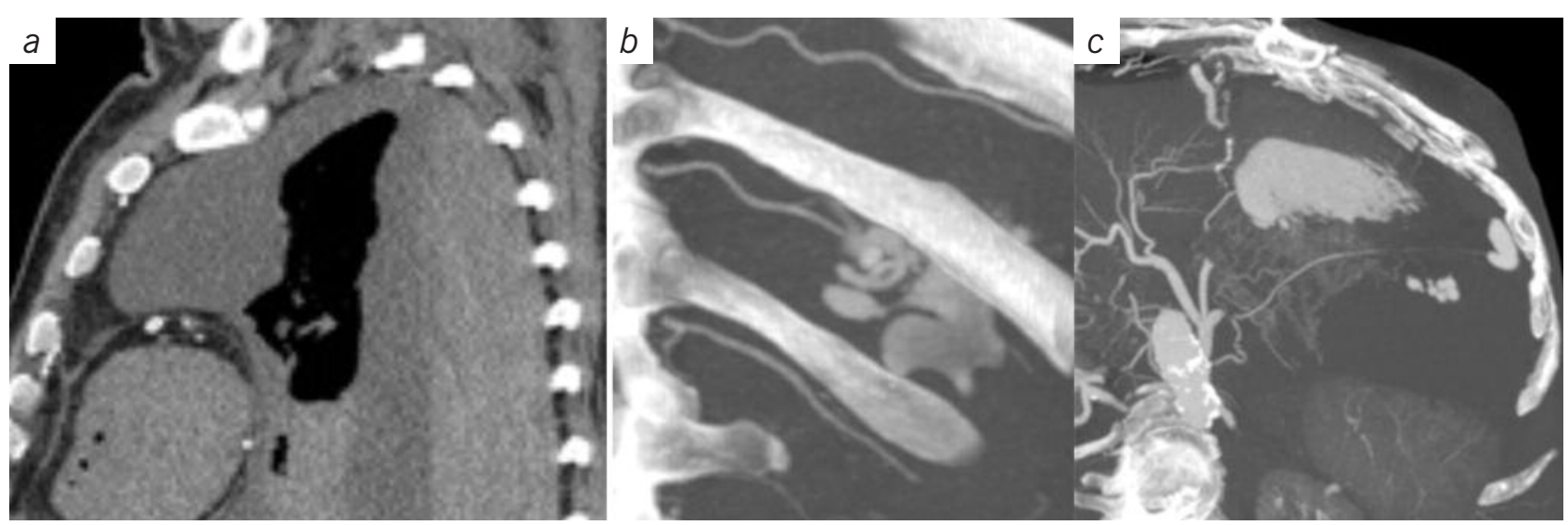

Fig. 4a-c. Bleeding into the mediastinum and into the chest. Multiple sources due to coagulationdisorder complicating bypass surgery.

a: Blood with layer formation (due to sedimentation) in the left chest.

$b$ : Contrast material extravasation from an intercostal artery on the left. The rib was fractured due to reanimation. MIP. PMCT-angiography.

c: Contrast material extravasation from the left $A$. diaphragmatica, possibly due to an accidental lesion by a puncture. MIP. PMCT-angiography.

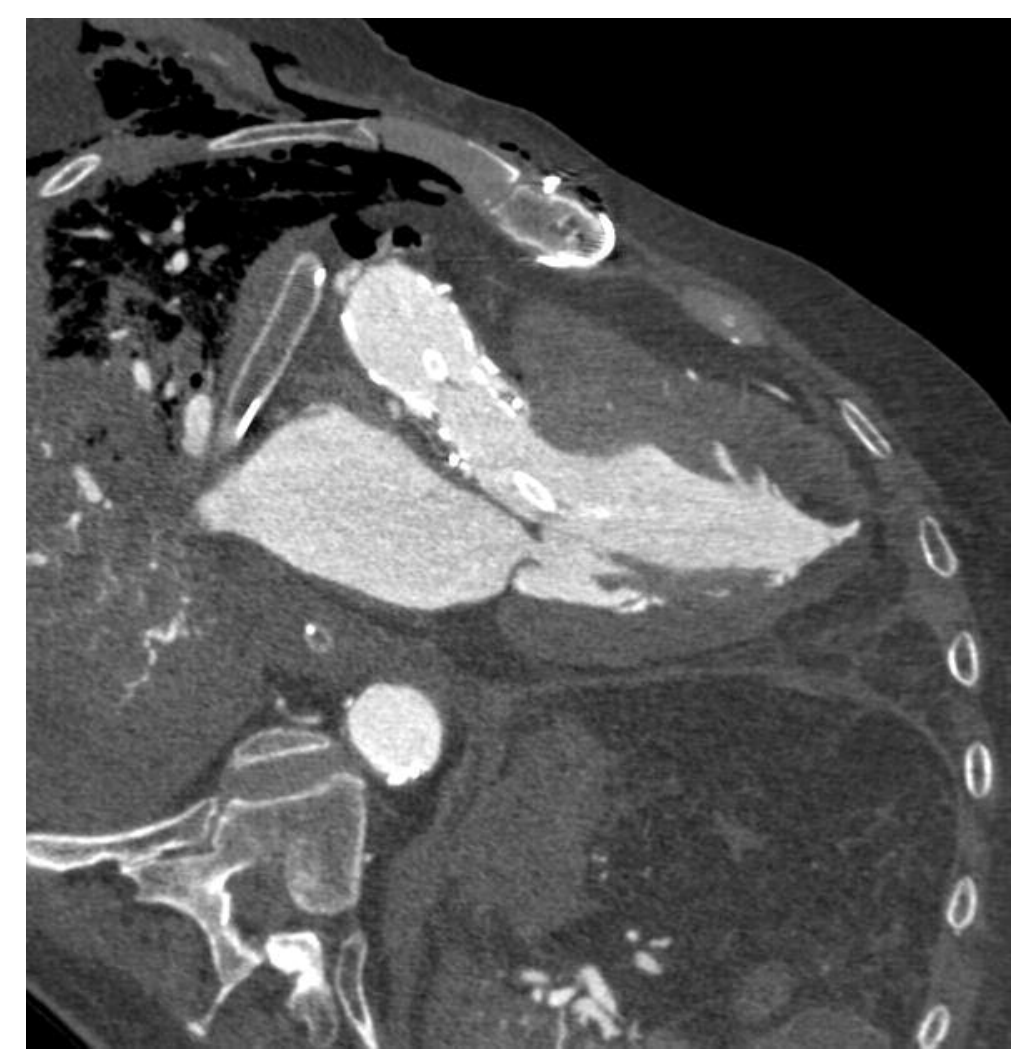

Fig. 5. Contrast material in the myocardium indicating the cardiotomy site during TAVI. LIMA bypass. PMCT-angiography. 


\section{Bypasses}

After bypass surgery, the bypass could also be analysed. PMCT visualised a contrasting bypass, and within the bypass calcifications, stents, and stents with further calcifications. LIMA (Left Internal Mammary Artery) bypass and RIMA (Right Internal Mammary Artery) bypass did show up by clips in typical formation. PMCT-angiography visualised the lumen of the bypass and of the anastomoses (Fig. 6a-c). It was difficult or even impossible to identify occluded bypasses without the help of calcified walls, check marks in the aortic wall, and characteristic clip distribution. This was true for both PMCT and also PMCT-angiography. An isolated observation showed such a bypass by virtue of contrast extravasation around it (Fig. 7h).

\section{Observation 1: Bleeding, Pneumothorax, Fracture of the Breastbone (Fig. 7a-h)}

The patient died shortly after heart surgery. PMCT showed blood in the mediastinum and in the left chest (Fig. 7a and f), and a bilateral pneumothorax with drainages (Fig. 7a and b); in the abdomen, an aneurysm of the abdominal aorta was visible as an incidental finding (Fig. 7f). Rib fractures showed a pattern typical for reanimation (fig. 7e). The chest compression had fractured the right half of the breastbone (Fig. 7c and d). An implanted aortic valve and stents in the RCX and the LAD were visible (Fig. 7a,e and f). Clips with a characteristic pattern indicated a LIMA bypass (Fig. 7a, e, and f).
PMCT-angiography showed the LIMA bypass filled with contrast material up to the apex of the heart (Fig. 7g). Within the contrast material, a bypass from the aorta to the RCA appeared (Fig. 7h). The bleeding had several sources. Therefore, a coagulation disorder was the most likely cause. The extravasation of the contrast material could be followed into the left and the right chest, the mediastinum and the pericardium (Fig. 7f-h).

\section{Implanted Valves}

It was easier to identify implanted valves when they contained metal - the more the better. Missing information about the type of the implanted valve made the analysis difficult. Knowing the type of the implanted valve permitted distinct evaluation of hitherto unknown complications recurring with this type of valve. Contrast from suture material and/or material placed to prevent bleeding oftentimes helped to recognise the type of the surgical procedure. This then allowed for a specific more precise analysis.

The analysis after TAVI may serve as an example of the search for typical modifications: in PMCT, the position of implanted valve in relation to the ostia of the coronary arteries is important (Fig. 8). PMCT-angiography demonstrates whether covering valves impede the blood flow. The lower margin of the implanted valve can modify the upper leaflet of the mitral valve and its suspension. PMCT raises suspicions; PMCT-angiography shows the modification (Fig. 9). Ruptures into the pericardium are
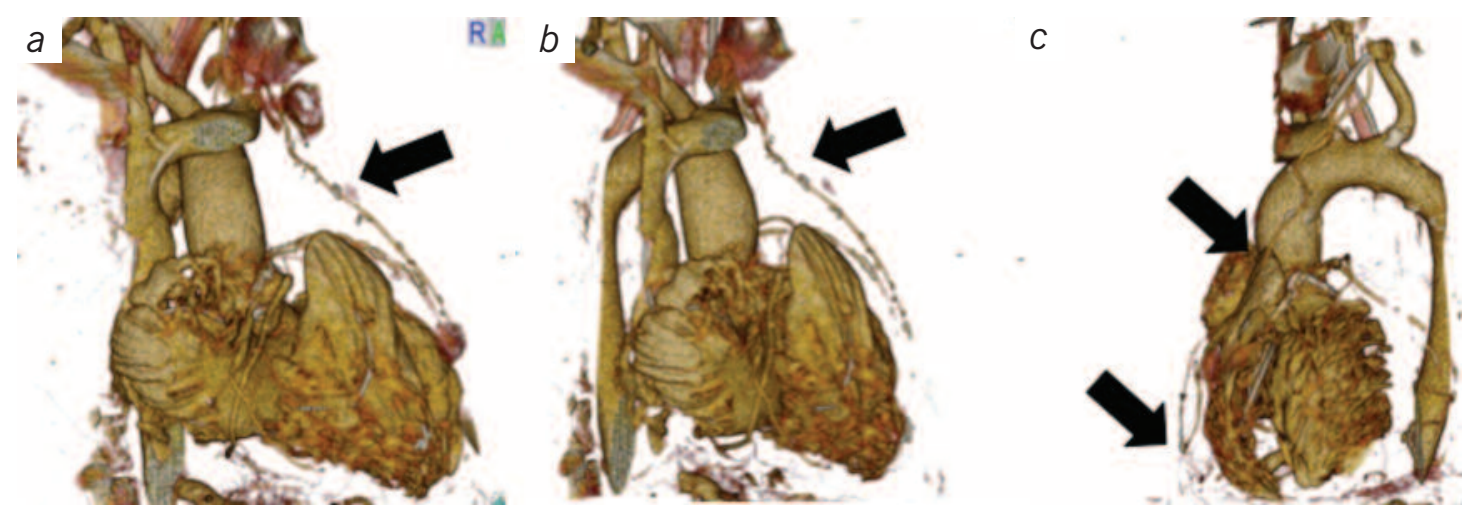

Fig. 6a-c. LIMA bypass (black arrow) to the LAD. The bypass is open. The irregularities of the Iumen of the LAD and the anastomosis indicate existing stenoses. PMCT-angiography, circulatory phase with filling of the right and the left heart, the aorta, the vena cava, and the coronary arteries 

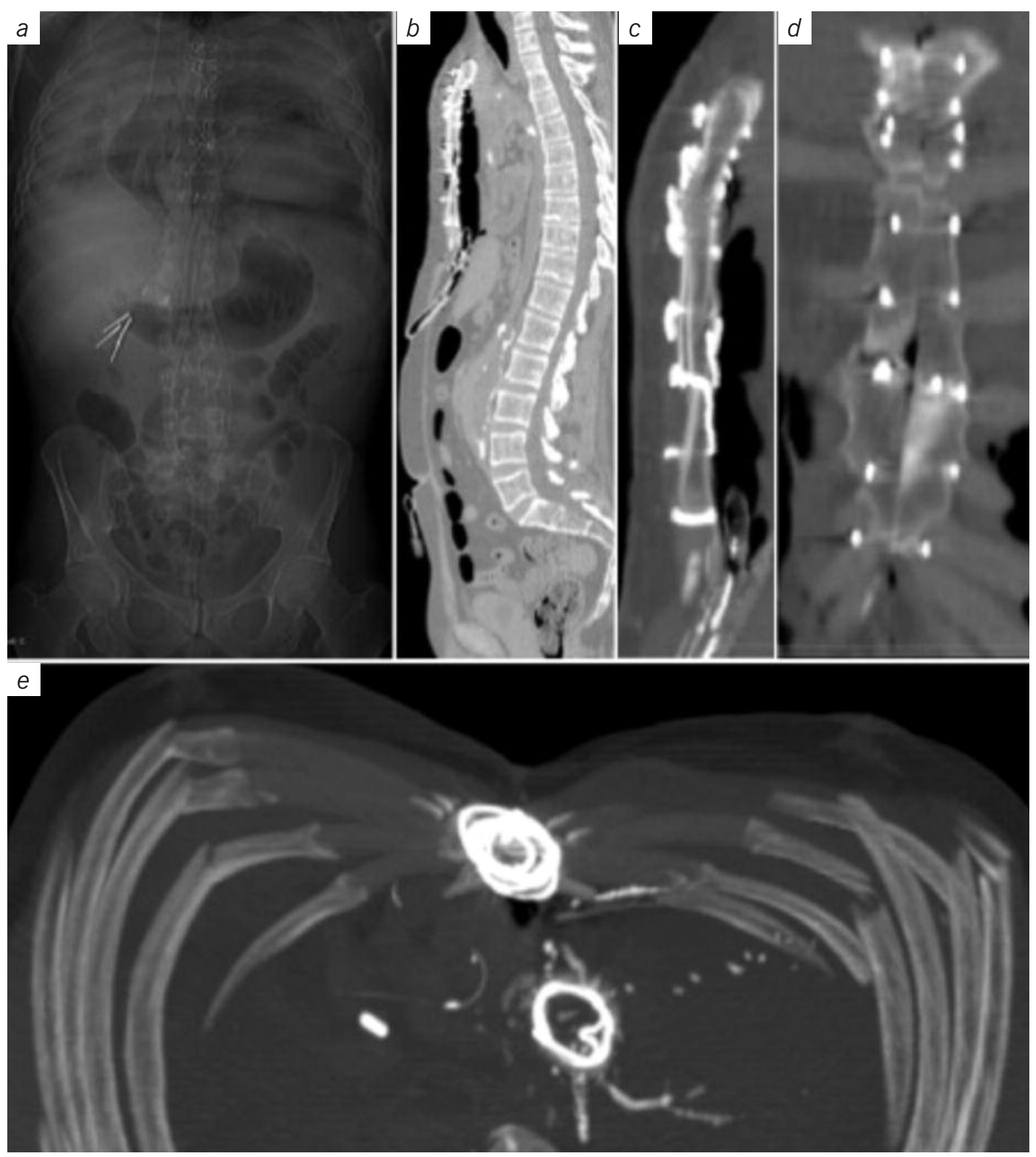

Fig. 7a-e. Bleeding during AVR (aortic valve replacement). PMCT.

$a$ and b: S/p AVR: Effusions, pneumothorax, catheter in the superior vena cava. Sutures in both groins (probably because of the attempt to place a vascular catheter). Sternotomy. Drainage in the mediastinum. PMCT. Topogram

c-e: Fractured ribs (due to reanimation), and fractured right half of the sternum (due to reanimation and to a wire-suture close to the osteotomy). Clips typical for a LIMA bypass. MIP (Maximum Intensity Projection) 

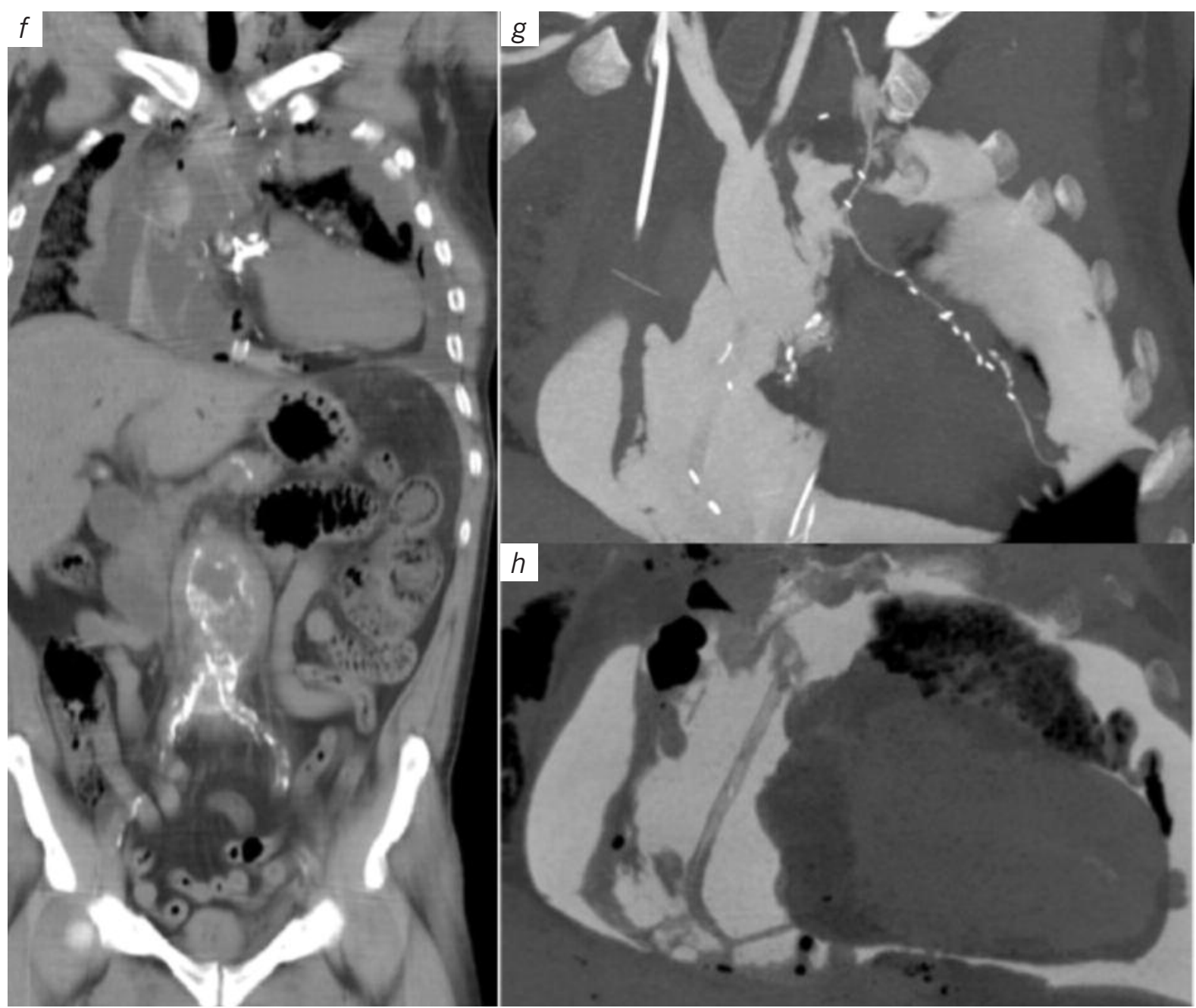

Fig. 7f-h. Fractured ribs (due to reanimation), and fractured right half of the sternum (due to reanimation and to a wire-suture close to the osteotomy). Clips typical for a LIMA bypass. MIP (Maximum Intensity Projection)

$g$ and $h$ : Contrast material extravasation into the pericardium, the pleural space and mediastinum. LIMA bypass to the LAD (filled with contrast material) and aorto-coronary bypass to the RCA (visible in the contrast extravasation - "lake" in the anterior mediastinum/pericardium). Catheter in the superior vena cava. PMCT-angiography, arterial phase. 


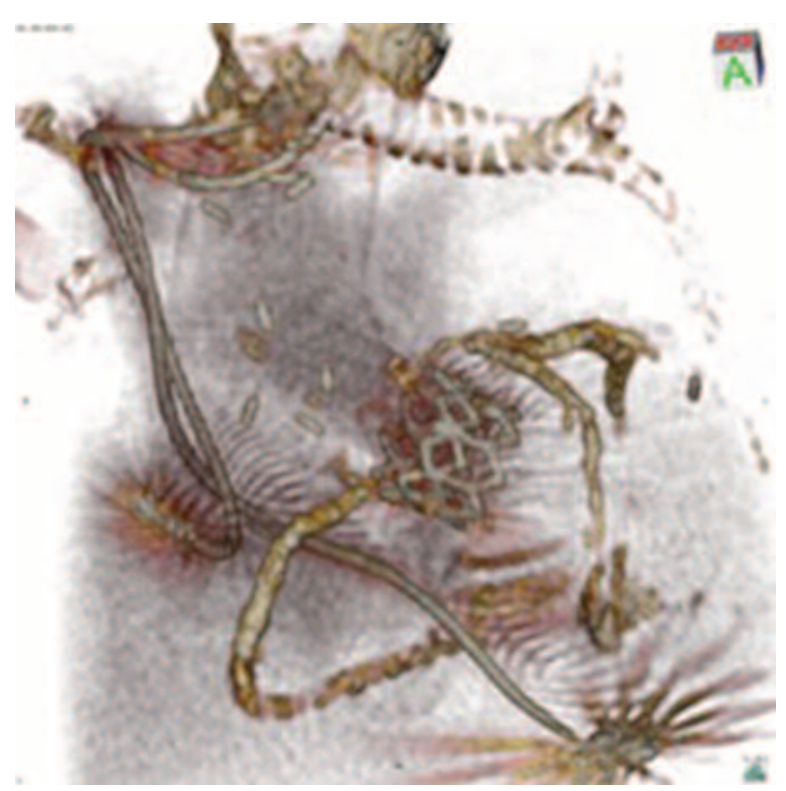

Fig. 8. The upper margin of the implanted aortic valve (Edwards Sapien valve) partially encroaches upon the ostia of the calcified coronary arteries. TAVI. Pacemaker. 3D reconstruction. PMCT.

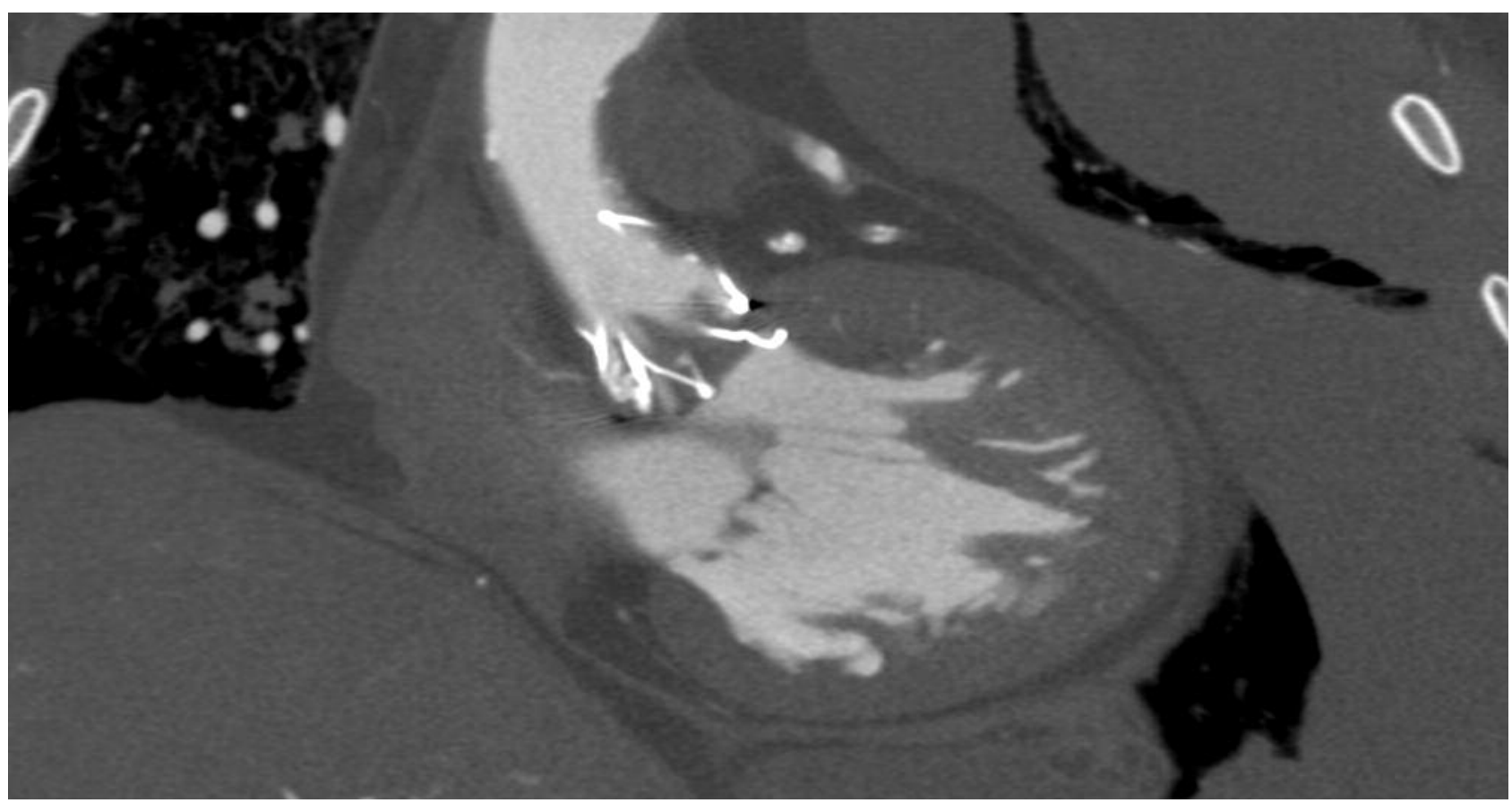

Fig. 9. The lower margin of the implanted aortic valve (JENA valve) involves the upper (anterior) insertion of yhe leaflet of the mitral valve and impairs its motility. TAVI. MIP. PMCT-angiography. 


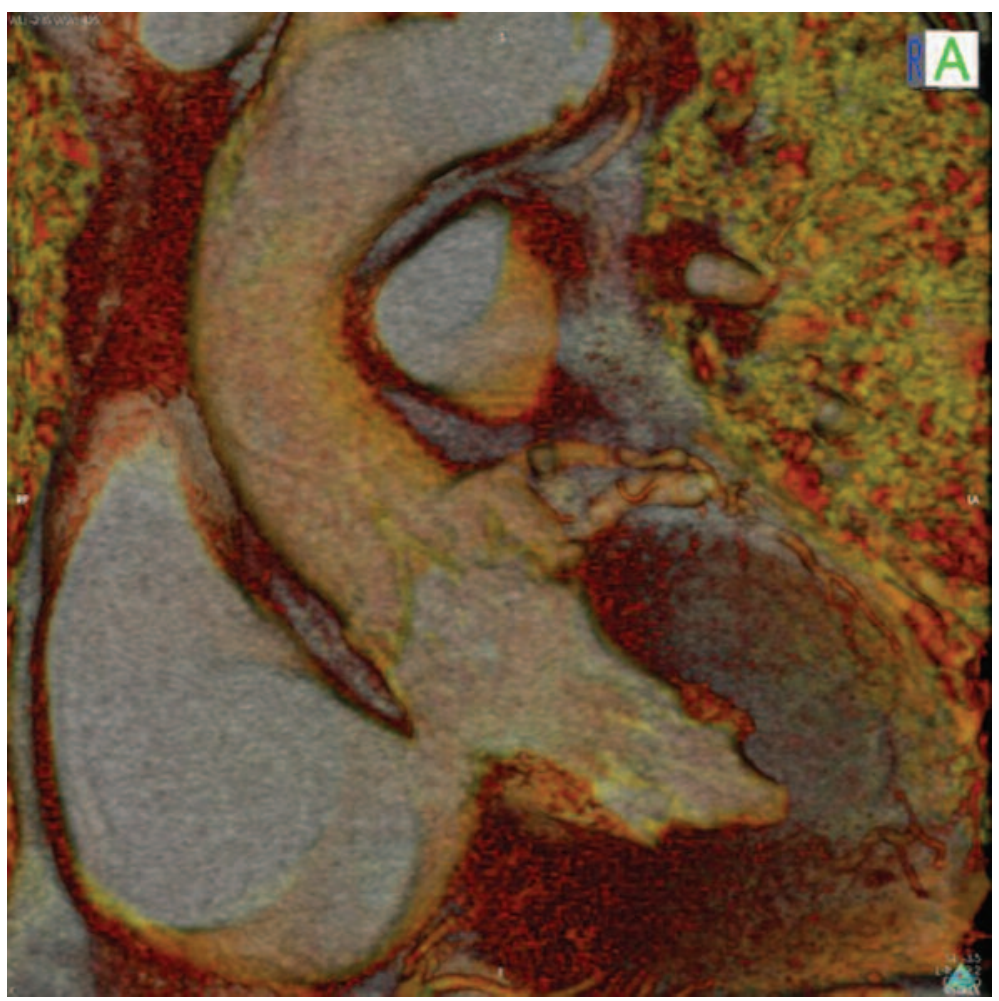

Fig. 10. Shunt from the left ventricle to the right atrium after TAVI (Ewards Sapien valve). 3D reconstruction. PMCT-angiography.

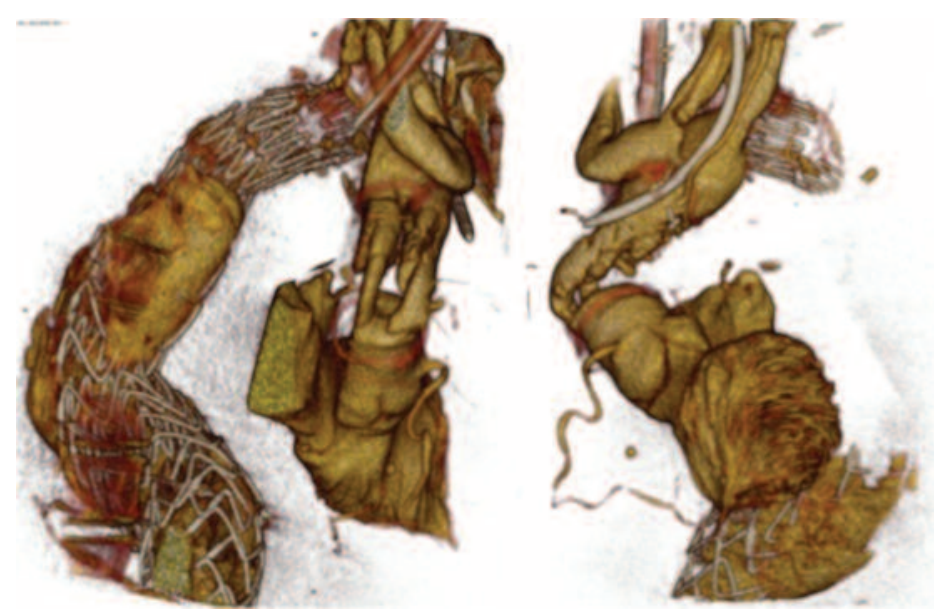

Fig. 11. "Chimney" anastomosis of the ascending aorta and stent in the descending aorta (after dissection). 3D reconstruction. PMCT-angiography. 
evident in PMCT. A shunt between the left ventricle and the right atrium due to a rupture during the dilatation is visible in PMCT angiography (Fig. 10).

The interpretation of the PMCT after complex surgery has even more limits when adequate information about the procedure is missing: Here the example is replacement of the ascending aorta and the aortic arch combined with a reinsertion of the three big aortic outlets. Compared to PMCT, PMCT-angiography has a greater potential (Fig. 11).

\section{Combination of Surgical Interventions}

The combination of different surgical interventions indicates possible postoperative complications. They may be typical: after cardiac surgery, a laparotomy could have been indicated when ischemia of the bowel was suspected. Hypotension of several hours is a possible cause. Often, PMCT may show an open abdomen and sometimes an open chest. Impaired motility of the bowel can be suspected when air-fluid-levels are visible in the loops of the small bowel and/or parts of the bowel have been resected and/or contrast material is found in the stomach or the upper bowel (applied for testing the passage). Fluid in the peritoneal space suggests peritonitis and/or failure of kidneys, heart and liver. General edema supports this interpretation. Renal insufficiency is probable when contrast material is visible in the renal parenchyma and signs of extra-renal excretion are present, such as contrast material in the gall bladder and in the small and large intestine.

\section{Observation 2: Heart Surgery and Abdominal Surgery (Fig. 12a-g)}

The patient died after heart surgery followed by abdominal surgery.

PMCT showed a pneumothorax with drainage. The drain was kinked, it ended in the gas containing pleural space (Fig. 12a and b). In the left cerebral hemisphere, there was an infarction. Bilateral pleural effusion and fluid in the lung parenchyma indicated cardiac insufficiency (Fig. 12a). There were bilateral fractures of ribs 3-7 with a pattern typical for reanimation, but no sternal fracture.

Prosthetic valve rings were visible in the position of the aortic valve and mitral valve (Fig. 12c). A stent with calcifications in the LAD and coronary sclerosis were apparent (Fig. 12c). The left ventricle was dilated (Fig. 12d).

The patient had a tracheal tube and also a catheter in the urinary bladder. Two central venous catheters inserted via the right and the left jugular vein ended both in the anonymous vein, respectively. There were catheters in the right and left femoral artery.

The abdomen was open (Fig. 12g). A bowel resection was not apparent. In the stomach, contrast material was visible, probably applied via a gastric tube to evaluate bowel transit (Fig. 12e). There were subhepatic and pelvic drains.

There was general edema, a small amount of free fluid the peritoneum and fluid in the scrotum. A contrasting aortic wall was attributed to ante-mortem contrast examination (Fig. 12f).

The PMCT findings suggest that after cardiac surgery, the blood pressure dropped and could not be raised for an extended period. One consequence (among potential others) was ischemia of the bowel. Laparotomy showed generalised ischemia of the bowel without any therapeutic options feasible.

\section{Postoperative Care}

During postoperative care, there were findings concerning tubes, catheters and drainage: gastric tubes ended in the oesophagus, they formed a loop in the stomach and ended in the esophagus, or they formed a loop in the larynx (Fig. 13 a and b).

The tracheal tube ended too high in the larynx and/or advanced into one main bronchus. Several times, air in the pleural space in spite of a pleural drainage was observed. A possible cause/explanations are: air entering after decoupling of the suction, kinking of the drainage (Fig. 12b), and impaired suction due to inadvertent entry into the lung parenchyma (Fig. 14a and b). With exception of the post-mortem decoupling, these causes could explain pleural effusion in spite of pleural drainage. Several drains in the chest indicated problems before death (Fig. 14a-c).

A loop of a pacemaker in the right ventricle with its lead in the right atrium was observed, too. This patient had had a TAVI. The diameter of the balloon of the tracheal tube measured more than $3 \mathrm{~cm}$ (Fig. 15). 


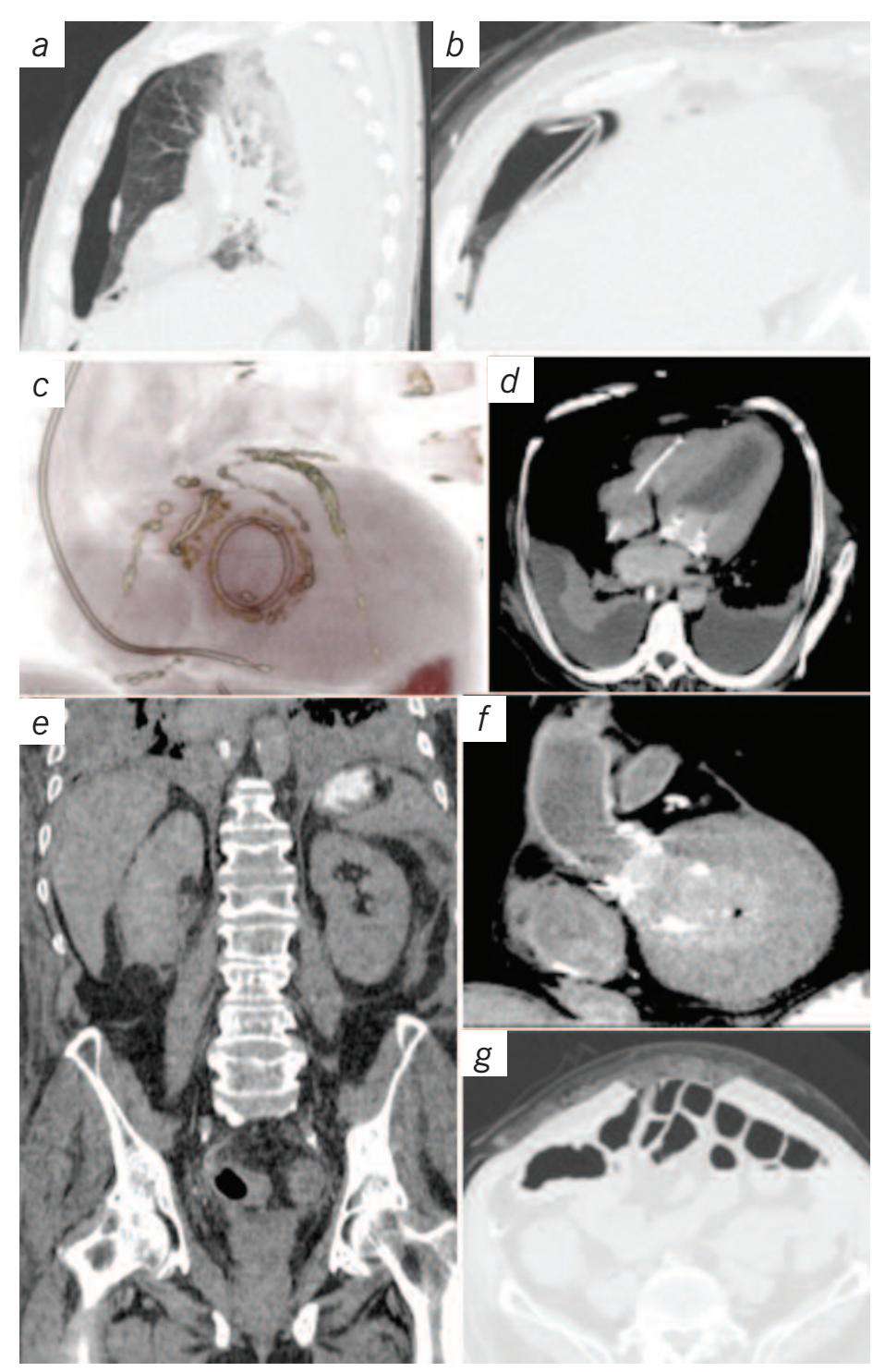

Fig. 12a-g. Cardiac surgery followed by abdominal surgery. PMCT.

a: Pneumothorax on the right. Fluid in the lungs with post mortem layering and pleural effusions: following ante-mortem cardiac insufficiency with pulmonary edema and pleural effusions.

b: Drainage in the pleural space filled with air, kinking of the drains, which could have caused impaired limited suction.

c: Implanted aortic valve and a metal ring at the basis of the mitral valve. Stent with calcification in the $L A D$. Calcifications of the coronary arteries. Pacemaker ending in the right ventricle. $3 D$ reconstruction.

$d$ : Dilated left ventricle. The pacemaker marks the (non-dilated) right ventricle. Oblique reconstruction (similar to a 4-chamber view).

e: Contrast material in the stomach, probably applied via a gastric tube for testing bowel transit.

f: Contrast-enhanced aortic wall attributed to diagnostic ante-mortem intravascular contrast material injection during CT diagnostic.

g: Open abdomen due to explorative laparotomy, probably due to suspected bowel ischemia. 

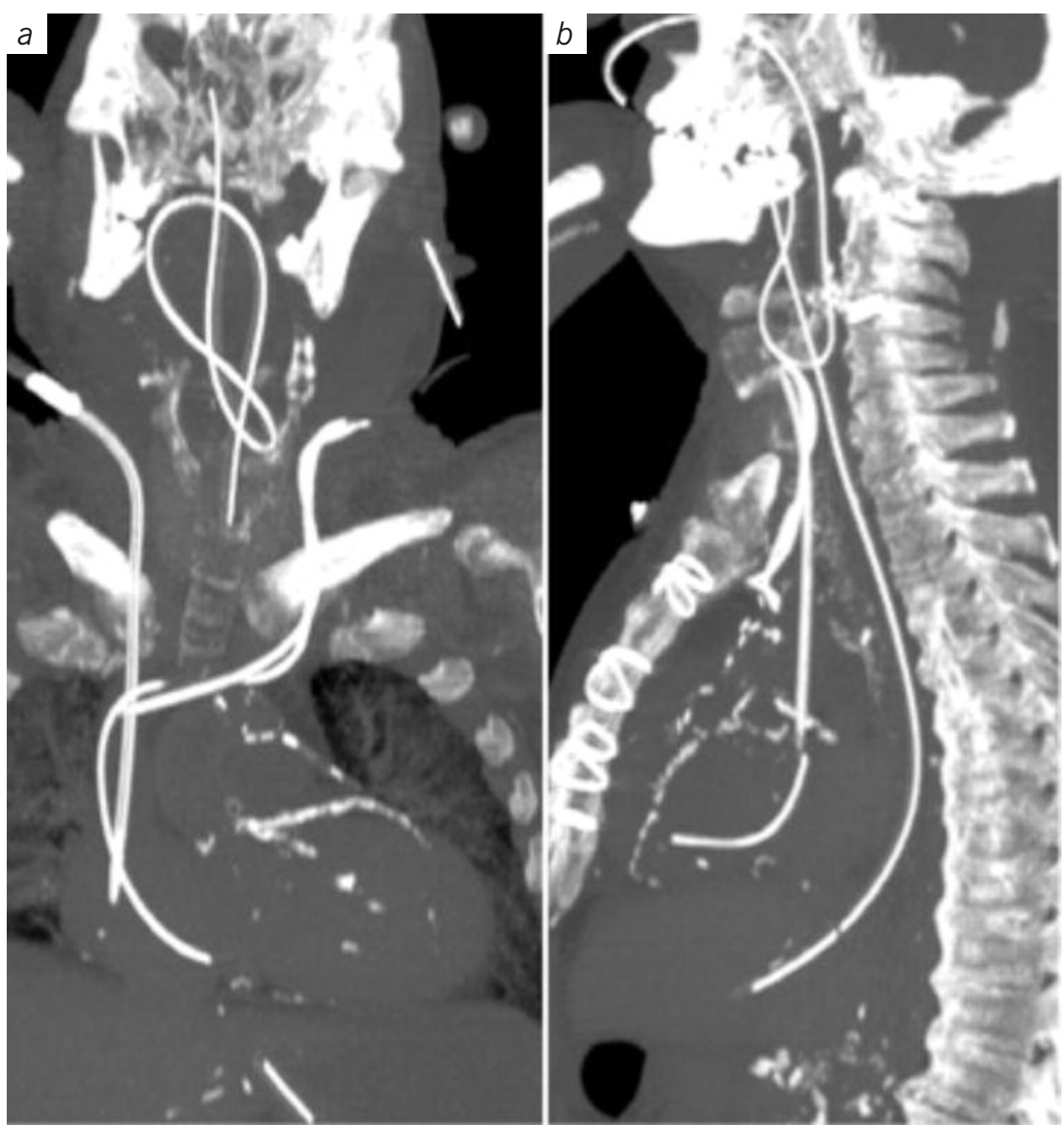

Fig. 13 a and b. Loop in the larynx. Bypass-surgery. PMCT, MIP. 


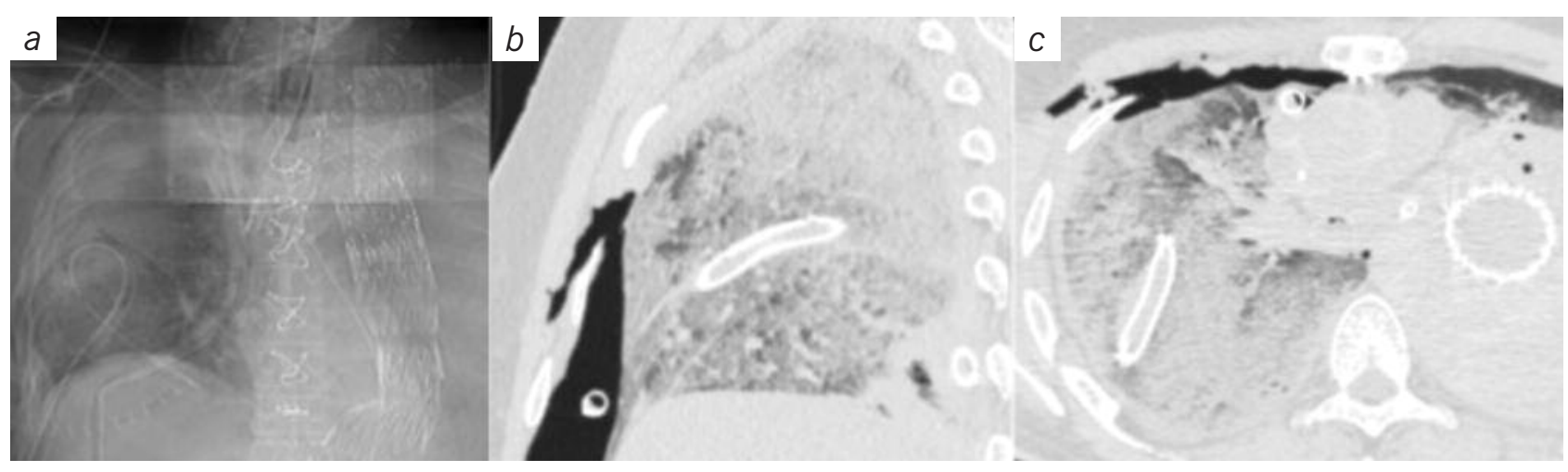

Fig. 14a-c. Two drains in the pleural space, one with kinking, the second with parenchymal entry. Pleural effusions. Pneumothorax (possibly after disconnecting the drains after death). Surgery because of dissection of the ascending aorta. Stent in the descending aorta. PMCT.

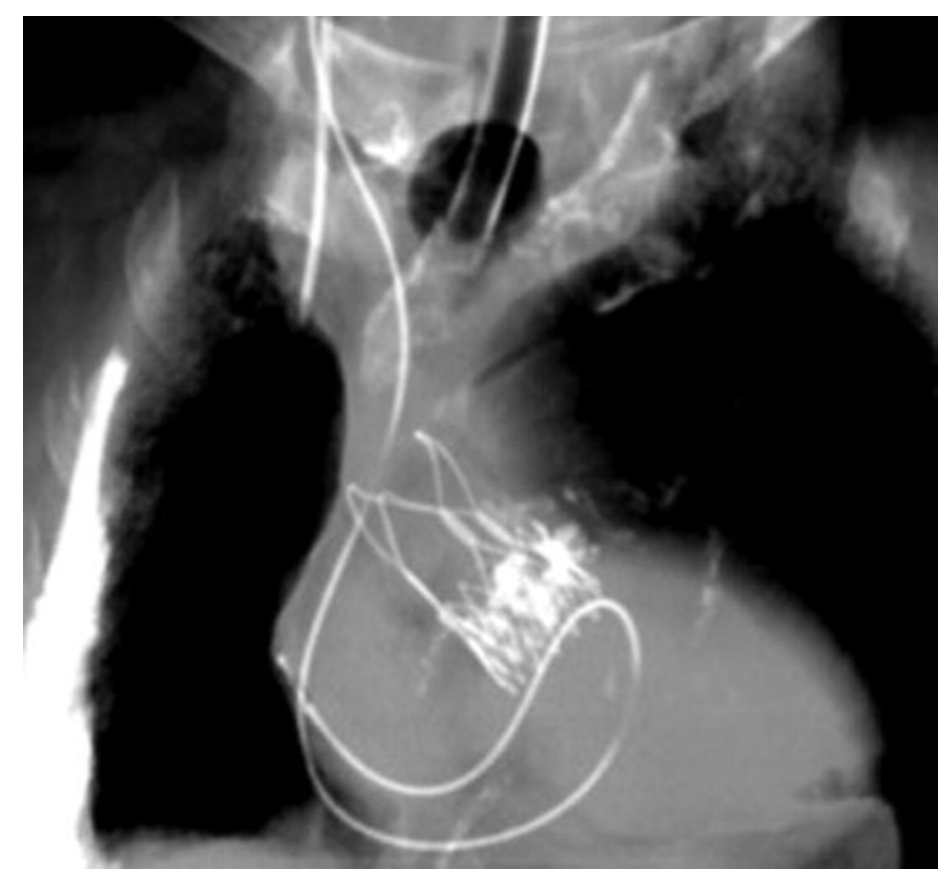

Fig. 15. Loop in the right ventricle of a pacemaker ending in the right atrium. Balloon of the tracheal tube measures more than $3 \mathrm{~cm}$ in diameter. TAVI. 


\section{DISCUSSION}

The above sections of the paper represent selected material, based on cases which have been recorded for different reasons. What these cases have in common is that they address findings after cardiac surgery and intensive post-op-care. It is not possible to indicate a relative frequency of complications. This remains a goal for future projects. The intention was to demonstrate the diagnostic value of PMCT and PMCT-angiography. Showing their diagnostic value necessitates demonstrating their limitations.

\section{Possibilities}

PMCT visualizes gastric and tracheal tubes, vascular catheters, drains and urinary catheters. Their tip, loops, kinking, and false tissue-passage are visible. The cause of an impaired function becomes obvious. These details can be documented systematically, which makes a difference to diagnostic imaging of the living: in the living, diagnostic imaging with ionizing radiation is limited by the necessity of radiation protection; diagnostic imaging has to answer questions. These questions concern diagnosis, therapeutic options, and prognosis. Quality control itself as a part of post-mortem examinations does not justify exposing a living person to ionising radiation. In many countries, the law even excludes quality control as a reason for any diagnostic imaging. PMCT has obviously the great advantage of unlimited radiation-exposure. Therefore, details can become apparent, which are not or but rarely seen in clinical radiology. Examples are fractures of the costal cartilage and the sternum after reanimation. PMCT-findings allow for discussing a potential procedural risk. Individuals involved in complications resulting from a surgical or post-op procedure can be identified and trained to avoid future complications. This training - also for groups - becomes an option.

PMCT can visualise the cause of death, like massive bleeding, cardiac tamponade, massive pleural effusions and large pneumothorax. There are combinations of PMCT findings suggesting cardiac insufficiency and multi-organ failure. However, these latter are deductions, which have o to be correlated with pre-existing clinical findings prior to surgery and concomitant death.
After heart surgery, PMCT visualises the metal of implanted valves. TAVI valves and their relation to the ostia of the coronary arteries and to the suspension of the mitral valve are visible. Suggestions about risks and possible failures are possible.

PMCT-angiography adds details not visible in PMCT. In bleeding, PMCT angiography visualizes the bleeding vessel. Extravasation of contrast material of several vessels indicates a coagulation disorder. After valve implantation, the function of the valve can be demonstrated. Partial stenosis and complete occlusion of coronary arteries become apparent. Shunts show up. Appropriate surgical handling can be proven and reasons for interventions become visible.

Compared to autopsy, PMCT and PMCT-angiography have advantages. The data can be analysed repeatedly, they can be transferred to third parties without delay - either by CT, USB stick, or the Internet. The data allow for a display in 3D, which facilitates their demonstration to third parties of lay people. Furthermore, PMCT and PMCT-angiography visualise details difficult or impossible to grasp during autopsy. The morphology of heart vessels, aorta and bypasses is displayed. The obtained images guide the autopsy, which allows for verification.

\section{Limits}

PMCT and PMCT-angiography have limits, not so much by the procedures themselves, but by the complete lack of or limited clinical information, what is a major disadvantage and often a real problem, which oftentimes is difficult or even impossible to overcome. There are common causes:

Surgery and post-op-manipulation: In case of the patient's death, physicians avoid describing their actions and their thinking. This is especially true when the result may have a potential legal implication. It is also very human to prefer a status of being successful rather than to be noted by complications and failures. This fact has to be respected and handled diplomatically and thus it may facilitate obtaining further information and/or recognising information as modified or selected.

Clinical findings and history: The knowledge of symptoms before death and of preceding diseases guides the analysis of PMCT and PMCT-angiogra- 
phy. Typical chest pains make it easier to classify the death as cardiogenic. Without further information, the existence of implanted valves and bypasses will tempt the radiologist to attribute death to heart disease.

Post-mortem rigor changes the shape of organs. Some aspects differ from those known in the radiology of the living: This is valid for the size of the heart. The statement that a heart is dilated or hypertrophic has to be made with care. Furthermore, after death, the fluid migration and intestinal gas formation change the pressure gradient in the chest. The diaphragm moves upwards. Both modify the aspect of the heart. The heart of a deceased has to be analysed with care: diagnostic imaging of the living is much different from diagnostic imaging of the deceased.

Known and unknown surgical procedures: It is easier to describe and to interpret the heart and the chest when the operation is known. This knowledge may turn a suggestion into a certainty. Given the knowledge of the type of the operation, one can search for signs of complications typical for the procedure. It is equally important to know whether other procedures have preceded the last operation in question. It would be ideal if this information were given directly. It becomes possible or at least much easier to distinguish between early and late complications, and between induced and underlying pre-existing morbidity.

Impairment of vital functions (respiration, blood pressure, urinary excretion) and failure of devices (suction, infusion, oxygenation) have causes. The knowledge of their existence would help the radiologist. Kinking of drains, loops of gastric tubes and blocked catheters are easy to demonstrate with PMCT and PMCT-angiography. The pathologist is able to find them. However, he has to look for them. If removed before autopsy, the position and the function of a tube or a catheter cannot be judged. Failed punctures can be the cause of pneumothorax or bleeding. A failed intubation can result in hypoxia of different organs. Multiple drains and multiple vascular catheters indicate problems in the postoperative care. We observed several deceased, who had six vascular catheters; all the individuals had a general edema. Acting under the impression that the catheters did not function adequately the staff in consequence inserted new catheters. How- ever, one had not been sure of their function either, therefore, all the catheters were left in place and infusions were given to keep them open. Thus, edema and weight gain resulted.

PMCT and PMCT-angiography do not replace histology. This fact has to be kept in mind when suspecting myocardial infarction. The radiologist does not see the myocardial infarction! He relies on indirect signs like impaired perfusion by stenosis or vascular obstruction in PMCT-angiography. A bleeding into the myocardium due to an infarction is an exception. This is of special importance when the blood supply of the heart is modified after bypass surgery: PMCT-angiography will show the changes in the diseased coronary arteries. Grabherr described contrast material-enhanced myocardium in PMCT-angiography and attributed this finding to an infarction [27]. We observed such an enhancement several times and found only once a myocardial infarction in this area. The significance of such an observation therefore remains an open question.

Selection: By definition, PMCT and PMCT-angiography is performed in the deceased. In such a group, findings that cause death or may cause death are expected to be seen more often than in living patients. It is even thinkable that findings, which cause death, are seen only in the deceased. Anyhow, post-mortem changes should not be observed in living persons. Furthermore, findings indicating complications/diseases compatible with survival should be seen more frequently in the living.

Clinical importance: PMCT and PMCT-angiography allow for distancing oneself from subjective perspectives. Individual interests can be minimized. The data can be analysed repeatedly. The digital data can be multiplied and transferred to others for evaluation. After submission of additional information and after new results of research, further analysis remains possible. The analysis is possible without the person actively involved in the procedure or post-op care. The analysis is not ascribed to one radiologist.

One can compare the configuration of implanted valves and the local anatomy, allowing for improved construction and placement. This is true for different devices. Regarding post-op-care, gastric and tracheal tubes, drainages, punctures and vascular catheters can be evaluated and training for future 
avoidance can be instituted or improved. The avoidance of misplacement and of failures of puncture saves lives or prevents postoperative suffering. The observations demonstrate that complications which endanger the survival of the patient shall be prevented.

\section{CONCLUSION}

PMCT and PMCT-angiography visualise the cause of death and complications. In the living, their recognition and consecutive avoidance means survival and/or less suffering for the patient. To know them allows for prevention. It is possible to develop measures that will improve future planning. PMCT and PMCT-angiography allow for the exclusion of faults and errors.

topsy in the diagnosis of adult deaths: a validation study. Lancet. 2012, 379: 136-142.

10. Okuda T., Shiotani S., Sakamoto N., Kobayashi T.: Background and current status of postmortem imaging in Japan: Short history of "Autopsy imaging (Ai)". Forensic Sci Int. 2013, 225: 3-8.

11. Kasahara S., Makino Y., Hayakawa M., Yajima D., Iti H., Iwase H.: Diagnosable and nondiagnosable causes of death by postmortem computed tomography: A review of 339 forensic cases. Leg Med. 2012, 14: 239-245.

12. Saunders S., Morgan B., Raj V., Rutty G.: Post-mortem computed tomography angiography: past, present and future. Forensic Sci Med Pathol. 2010, 7: 271-277.

13. Grabherr S., Djonov V., Yen K., Thali M. J., Dirnhofer R.: Postmortem angiography: Review of former and current methods. AJR. 2007, 188: 832-838.

14. Jackowski C., Thali M., Sonnenschein M., et al. Virtopsy: postmortem minimally invasive angiography using cross section techniques - implementation and preliminary results. J Forensic Sci. 2005, 50: 1175-1186.

15. Jackowski C., Bolliger S., Aghayev E., Christe A., Kilchoer T., Aebi B., Périnat T., Dirnhofer R.: Reduction of postmortem angiography-induced tissue edema by using polyethylene glycol as a contrast agent dissolver. J Forensic Sci. 2006, 5: 1134-1137.

16. Grabherr S., Djonov V., Friess A., Thali M. J., Ranner G., Vock P., Dirnhofer R.: Postmortem angiography after vascular perfusion with diesel oil and a lipophilic contrast agent. AJR. 2006, 187: W515-W523. 
17. Grabherr S., Gygax E., Sollberger B., Ross S., Oesterhelweg L., Bolliger S., Christe A., Djonov V., Thali M. J., Dirnhofer R.: Two-step post-mortem angiography with a modified heart-lung machine: preliminary results. AJR. 2008, 190: 345-351.

18. Jolibert M., Cohen F., Bartoli C., Boval C., Vidal V., Gaubert J. Y., Moulin G., Petit P., Bartoli J. M., Leonetti G., Gorincour G.: Postmortem CT-angiography: feasibility of US-guided vascular access. J Radiol. 2011, 92: 446-449.

19. Sakamoto N., Senoo S., Kamimura Y., Uemura K.: Case report: cardiopulmonary arrest on arrival case which underwent contrast-enhanced postmortem CT. Journal of Japanese Association for Acute Medicine. 2009, 30: 114-115.

20. Lizuka K., Sakamoto N., Kawasaki H., Miyoshi T., Komatsuzaki A., Kikuchi S.: Usefulness of contrast-enhanced postmortem CT. Innervision. 2009, 24: 89-92.

21. Saunders S. L., Morgan B., Raj V., Robinson C. E., Rutty G. N.: Targeted post-mortem computed tomography cardiac angiography: proof of concept. Int J Legal Med. 2011, 125: 609-616.

22. Roberts I. S., Benamore R. E., Peebles C., Roobottom C., Traill Z. C.: Diagnosis of coronary artery disease using minimally invasive autopsy: evaluation of a novel method of post-mortem coronary CT angiography. Clin Radiol. 2011, 66: 645$-650$.

23. Morgan B., Biggs M. J., Barber J., Raj V., Amoroso J., Hollingbury F. E., Robinson C., Rutty G. N.: Accuracy of targeted post-mortem computed tomography coronary angiography compared to assessment of serial histological sections. Int J Legal Med. 2013, 127: 809-817.

24. Robinson C., Barber J., Amoroso J., Morgan B., Rutty G.: Pump injector system applied to targeted post-mortem coronary artery angiography. Int J Legal Med. 2013, 127: 661-666.

25. Wichmann D., Obbelode F., Vogel H., Höpker H., Püschel K., Kluge S.: Virtual autopsy as an alternative to traditional medical autopsy: a prospective cohort study. Ann Intern Med. 2012, 156 : 123-130.

26. Freres M., Walter C.: Behandlungsfehler im Krankenhaus. Offenlegen - entschuldigen - entschädigen. Deutsches Ärzteblatt. 2013, 1632-1634.

27. Graherr S.: Advances in post-mortem CTangiography. 2012, IALM, $6^{\text {th }}$ July. Oral communication.

Address for correspondence:

Prof. Dr. H. Vogel

Institute for Legal Medicine University Hospital Eppendorf

Butenfeld 34

22529 Hamburg

e-mail: Hermann.vogel@gmx.de 